\title{
Locally Enhanced Angiogenesis Promotes Transplanted Cell Survival
}

\author{
MOLLY K. SMITH, M.S., 1 ,* MARTIN C. PETERS, Ph.D., 2,* \\ THOMAS P. RICHARDSON, Ph.D., ${ }^{2,3}$ JESSICA C. GARBERN, B.S., ${ }^{1}$ \\ and DAVID J. MOONEY, Ph.D. ${ }^{1-3}$
}

\begin{abstract}
A developing therapy for complete or partial loss of function in various tissues and organs involves transplanting an appropriate cell population, capable of compensating for the existing deficiencies. Clinical application of this type of strategy is currently limited by the death or dedifferentiation of the transplanted cells after delivery to the recipient. A delay in thorough vascularization of the implant area creates an environment low in oxygen and other nutrients, and likely contributes to the initial death of transplanted cells. We have addressed this problem by sustained delivery of vascular endothelial growth factor (VEGF), an initiator of angiogenesis, from a porous polymer matrix utilized simultaneously for cell delivery. As expected from previous studies, VEGF delivered from these constructs elicited an enhanced angiogenic response over a 2-week period when implanted subcutaneously in SCID mice. Hepatocytes implanted using VEGF-containing matrices demonstrated significantly greater survival after 1 week in vivo as compared with cells implanted on matrices without growth factor. The results of this study therefore indicate that enhancing vascularization in the location of transplanted cells promotes their survival. In addition, this delivery system may be used in future studies to directly promote cell survival and function by also providing growth factors specific to the transplanted cells.
\end{abstract}

\section{INTRODUCTION}

$\mathbf{T}$ Ransplantation of selected cell populations is currently being investigated to regenerate and engineer a number of tissues in the body. In tissue engineering and related therapeutic strategies, cells are often delivered within three-dimensional constructs designed to inform cell function and/or overall tissue shape, as well as provide space for efficient delivery of an appropriate cell number. However, clinical application of cell-based therapies is limited, as the transplanted cells often lose function or die after delivery to the recipient and this is especially notable in the case of cells with high metabolic activity, such as hepatocytes. ${ }^{1,2}$ The massive cell loss that occurs within several days of transplantation is likely due to inadequate availability of nutrients for cells in the interior of delivery devices. Oxygen supply is particularly limiting, as cells more than approximately $200 \mu \mathrm{m}$ from a blood vessel suffer from hypoxia and die. ${ }^{3,4}$ The vasculature of the host will eventually penetrate macroporous matrices utilized for cell delivery via the process of angiogenesis, by which new blood vessels sprout from those that already exist and grow into undervascularized regions nearby. This natural angiogenic response is, however, insufficient to support the larger cell masses required to provide therapeutic support, and therefore likely

\footnotetext{
${ }^{1}$ Departments of Chemical Engineering, ${ }^{2}$ Biomedical Engineering, and ${ }^{3}$ Biologic \& Materials Sciences, University of Michigan, Ann Arbor, Michigan.

*These authors contributed equally to this work.
} 
contributes to the limited applicability of these applications.

Extensive studies of the angiogenic process have yielded knowledge of the identity and function of critical molecules involved in this phenomenon, making it possible to manipulate blood vessel sprouting through delivery of appropriate factors. One of the key regulators of angiogenesis is vascular endothelial growth factor (VEGF), a potent endothelial cell-specific mitogen and chemoattractant $t^{5}$ produced by a variety of cell types in response to low oxygen levels. ${ }^{6}$ A number of studies focusing on VEGF, both in vitro and in vivo, have elucidated mechanisms through which VEGF induces blood vessel sprouting. ${ }^{6,7}$ It has also been found that defects in spatial or temporal regulation of VEGF production are associated with pathological conditions (e.g., cancerous tumor growth, disorders of retina vascularization), indicating the importance of delivering this molecule only where and when it is needed in therapeutic applications. ${ }^{8}$ Other important factors to consider when delivering VEGF are its heparin-binding property and its short halflife in the body, because these will largely determine the area over which delivered growth factor may elicit a response. Together, these characteristics suggest that controlled, localized delivery may be the best approach to enhance vascularization when utilizing VEGF. With this type of strategy it is possible to address spatial and temporal requirements, as well as growth factor stability, because it may be protected from denaturation within the delivery device. Successful delivery of bioactive molecules from polymer constructs has been demonstrated for a variety of growth factors, ${ }^{9-13}$ and studies looking specifically at VEGF have demonstrated improved vascularization of the delivery construct. ${ }^{14-16}$

In the present study, we hypothesized that delivery of VEGF from a polymer scaffold utilized to simultaneously deliver a cell population would speed vascularization of the construct, and therefore enhance cell survival. Hepatocytes were selected as a model cell type because they are desirable for tissue-engineering applications ${ }^{2,17}$ but difficult to effectively transplant. ${ }^{1,18}$ The native architecture of the liver is such that hepatocytes are rarely observed more than two cell diameters from a functional blood vessel, emphasizing their high level of metabolic activity and particular dependence on transport of oxygen and other nutrients. Enhancing angiogenesis and improving the environment around the transplanted hepatocytes may therefore promote their survival after transplantation.

\section{MATERIALS AND METHODS}

\section{Matrix fabrication}

Scaffolds were formed by a modification of a previously published process, ${ }^{19,20}$ which involves fabricating poly(lactide-co-glycolide) (PLG) into microspheres before forming scaffolds from the polymer. Microspheres were made with 85:15 PLG (Resomer RG858; Boehringer Ingleheim; Ingleheim, Germany) via a standard doubleemulsion process. ${ }^{21}$ In brief, a 5\% (w/v) solution of PLG in ethyl acetate (Sigma, St. Louis, MO) was combined with distilled water in a test tube, and this two-phase mixture was sonicated (Vibracell; Sonics \& Materials, Newtown, CT) to yield a single emulsion. An equal volume of an aqueous solution containing $1 \%$ poly(vinyl alcohol) (PVA, MW 25,000, 88\% hydrolyzed; Polysciences, Warrington, PA) and 7\% ethyl acetate was added to the single emulsion, and the resulting solution was vortexed (Vortex Genie; VWR, West Chester, PA) to yield the double emulsion. This double emulsion was immediately transferred to $200 \mathrm{~mL}$ of a rapidly stirring aqueous solution of $0.3 \%$ PVA-7\% ethyl acetate. After allowing the ethyl acetate to evaporate over a 3-h period, the microsphere-containing solution was filtered to retrieve the microspheres, which were then rinsed with water and lyophilized.

For each matrix, $3 \mathrm{mg}$ of microspheres was combined with $10 \mu \mathrm{g}$ of vascular endothelial growth factor (VEGF) (Intergen, Purchase, NY) dissolved in $150 \mu \mathrm{L}$ of a $0.1 \%$ MVM alginate (Pronova, Oslo, Norway) solution. This mixture was lyophilized to form a powder, combined with $50 \mathrm{mg}$ of $\mathrm{NaCl}$ (sieved to yield a particle diameter between 250 and $425 \mu \mathrm{m}$ ), and pressed in a $4.76-\mathrm{mm}$-diameter die to create a 1.5 -mm-thick disk. The PLG was gas foamed by subjecting the disks to $\mathrm{CO}_{2}\left(800 \mathrm{lb} / \mathrm{in}^{2}\right)$ for $24 \mathrm{~h}$, followed by a rapid reduction of pressure to ambient. The $\mathrm{NaCl}$ was leached from matrices by incubation in $0.1 \mathrm{M} \mathrm{CaCl}_{2}$ for $16 \mathrm{~h}$ before use in experiments. Blank control matrices were manufactured by omission of VEGF from the processing protocol.

\section{Matrix characterization}

The size and volume distribution of microspheres were measured with a ZM Coulter counter (Beckman Coulter, Fullerton, CA). Imaging of representative microspheres and porous matrices was performed with a scanning electron microscope (ISI-DS 130; Topcon Technologies, Pleasanton, CA) on samples that were dried and sputter coated with gold (Desk II; Denton Vacuum, Cherry Hill, $\mathrm{NJ})$.

\section{VEGF release kinetics}

To determine the incorporation efficiency and release kinetics of VEGF from the porous matrices, receptorgrade ${ }^{125}$ I-labeled human VEGF $(100 \mu \mathrm{Ci} / \mu \mathrm{g}$ ) (Biomedical Technologies, Stoughton, MA) was utilized as a tracer. Approximately $1 \mu \mathrm{Ci}$ of labeled VEGF was incorporated into each matrix, along with the $10 \mu \mathrm{g}$ of unlabeled VEGF as described above. To determine the re- 
lease of VEGF, six matrices prepared with the radiolabeled VEGF were placed in $3 \mathrm{~mL}$ of phosphate-buffered saline (PBS; GIBCO, Grand Island, NY) and maintained at $37^{\circ} \mathrm{C}$. At various times, the matrices were removed from the PBS, and the radioactivity remaining in each matrix was measured with a $\gamma$ counter. The amount of VEGF released from the matrices was determined at each time point by counting the removed samples in a $\gamma$ counter and comparing the result with the total ${ }^{125} \mathrm{I}-1 \mathrm{a}-$ beled VEGF measured in the matrices before they were placed in PBS.

\section{Hepatocyte isolation}

Hepatocytes were isolated from adult male Lewis rats (150-200 g; Charles River Laboratories, Wilmington, MA) by a modification of the original Seglen two-step collagenase perfusion procedure. ${ }^{22}$ Briefly, the liver was perfused via the portal vein, first with calcium-free buffer (142 mM NaCl, $7 \mathrm{mM} \mathrm{KCl,} 20 \mathrm{mM}$ HEPES, $\mathrm{pH}$ $7.4,37^{\circ} \mathrm{C}$ ) for $10 \mathrm{~min}$, followed immediately by collagenase-containing buffer $(142 \mathrm{mM} \mathrm{NaCl}, 7 \mathrm{mM} \mathrm{KCl}$, $20 \mathrm{mM} \mathrm{CaCl}_{2}, 20 \mathrm{mM}$ HEPES, collagenase type $2[0.5$ $\mathrm{mg} / \mathrm{mL}$; Worthington Biochemical, Lakewood, NJ], $\mathrm{pH}$ $\left.7.4,37^{\circ} \mathrm{C}\right)$. Perfusion with the collagenase-containing buffer was continued for 7-10 min, until the liver tissue was sufficiently disrupted as determined by visual inspection. The perfused liver was then placed in icecold William's Medium E, and dissociation of the cells was performed by removing the liver capsule and gently shaking the hepatocytes free from the remaining liver matrix. This cell suspension was then filtered through a 400- $\mu$ m Nytex mesh (Sefar America, Tetko, Depew, NY) to remove large debris. The cell yield per liver was $1-2 \times 10^{8}$ and the initial percentage of viable cells was $80-90 \%$. To further purify the cell population and remove cellular debris, density gradient centrifugation using Percoll (Sigma) was performed. The collected hepatocytes, which had a total cell viability greater than $90 \%$, were then placed on ice and immediately used in experiments.

\section{SCID mouse implants}

Matrices $(n=3)$ from each condition were implanted in subcutaneous pockets (two per animal) on the dorsal region of 7- to 9-week-old male SCID mice (cb17/SCID) (Taconic, Germantown, NY). A 1.5-cm longitudinal incision was made on each animal's back and one implant was then placed between the skin and muscle layers on either side of the incision. The incision was closed with two interrupted sutures and covered with tissue adhesive (Mastisol; Ferndale Laboratories, Ferndale, MI) to help seal the opening. All National Institutes of Health (NIH, Bethesda, MD) guidelines for the care and use of laboratory animals were observed. ${ }^{22 a}$
For conditions including transplanted cells, immediately before matrix implantation $1 \times 10^{6}$ hepatocytes were suspended in $25 \mu \mathrm{L}$ of a 1:1 mixture of William's Medium E and growth factor-reduced Matrigel (BD Biosciences Discovery Labware, Bedford, MA) and allowed to absorb into the polymer matrix for $5 \mathrm{~min}$. The cellseeding efficiency was determined by counting the cells remaining in the dish after this absorption period.

Mice were killed and matrices retrieved at 7 and 14 days for vascularization studies, and at 3 and 7 days for hepatocyte studies. The matrices were dissected out of the tissue and immediately placed into $10 \mathrm{~mL}$ of buffered zinc-formalin, where they remained for $24 \mathrm{~h}$ at $4^{\circ} \mathrm{C}$. The zinc-formalin was then exchanged for $70 \%$ ethanol and samples were stored at $4^{\circ} \mathrm{C}$ until they were embedded in paraffin by the University of Michigan, School of Dentistry, Histology Core (Ann Arbor, MI).

\section{Histology staining}

Paraffin-embedded matrices were cut into $5-\mu \mathrm{m}$ sections and placed on glass slides for histological analysis. Tissue sections were either stained with Gill's 3 hematoxylin (Sigma) and aqueous eosin Y solution (Sigma) to visualize overall tissue morphology, or immunostained with antibodies raised against mouse CD31 (clone MEC 13.3) (BD Biosciences Pharmingen, San Diego, CA) to detect endothelial cells and mouse vasculature.

To immunostain tissue sections, the slides were first deparaffinized and rehydrated, and then washed in $3 \%$ $\mathrm{H}_{2} \mathrm{O}_{2}$ (Sigma) in PBS to quench endogenous peroxidase activity. To improve antigen exposure the slides were treated with citrate buffer (Biocare Medical, Walnut Creek, CA). Nonspecific antibody interactions were blocked by incubating the slides in Terminator blocking solution (Biocare Medical) for $10 \mathrm{~min}$ at $25^{\circ} \mathrm{C}$. Antimouse CD31 primary antibody, diluted in Van Gogh Green antibody diluent (Biocare Medical), was then incubated with the sections for $1.5 \mathrm{~h}$ at $25^{\circ} \mathrm{C}$. Bound primary antibody was then detected with a biotinylated secondary antibody, followed by a streptavidin-horseradish peroxidase (HRP) conjugate (both from Biocare Medical). The substrate added for the HRP reaction was 3,3'diaminobenzidine (DAB) (Zymed, South San Francisco, CA). Finally, sections were lightly counterstained with hematoxylin.

\section{Histological analysis}

Tissue sections were visualized and photographed with an E-800 light microscope (Nikon, Melville, NY). Three samples from each condition, with six sections from each sample, were analyzed manually and digitally with NIH Image software (developed by the U.S. National Institutes of Health). Blood vessels present in the implants were analyzed for their total number, and were identified 
by their defined lumens in CD31-stained tissues at $\times 200$ magnification.

For conditions utilizing transplanted hepatocytes, the total number of engrafted hepatocytes in each section was manually counted at $\times 200$ magnification. Engrafted hepatocytes were identified by their large size, large and spherical nuclei, and distinct cytoplasmic staining as described in a number of published reports. ${ }^{2,23,24}$

\section{Statistical analysis}

The Student $t$ test (one-tailed) was utilized to analyze the statistical significance of differences between data from VEGF and control conditions for both blood vessel and engrafted hepatocyte quantification, with $p \leq 0.05$ taken as significant. InStat version 2.01 software (GraphPad Software, San Diego, CA) was utilized to perform this analysis.

\section{RESULTS}

\section{Matrix characterization}

Microspheres fabricated for use in matrix preparation (Fig. 1A) had a number average diameter of $2.3 \mu \mathrm{m}$, and a volume average diameter of $20 \mu \mathrm{m}$ (Fig 1B). Matrices formed with microspheres had an interconnected open porous structure (Fig. 2A). The efficiency of VEGF incorporation was measured to be $36 \pm 1 \%$. In vitro, this system exhibits a release profile in which $25 \%$ of the incorporated growth factor is released within 3 days, followed by a subsequent constant release of approximately $1 \% /$ day for the duration of the study (Fig. 2B).

\section{Implant vascularization}

Matrices processed to release VEGF, and control matrices containing no growth factor, were implanted into

A
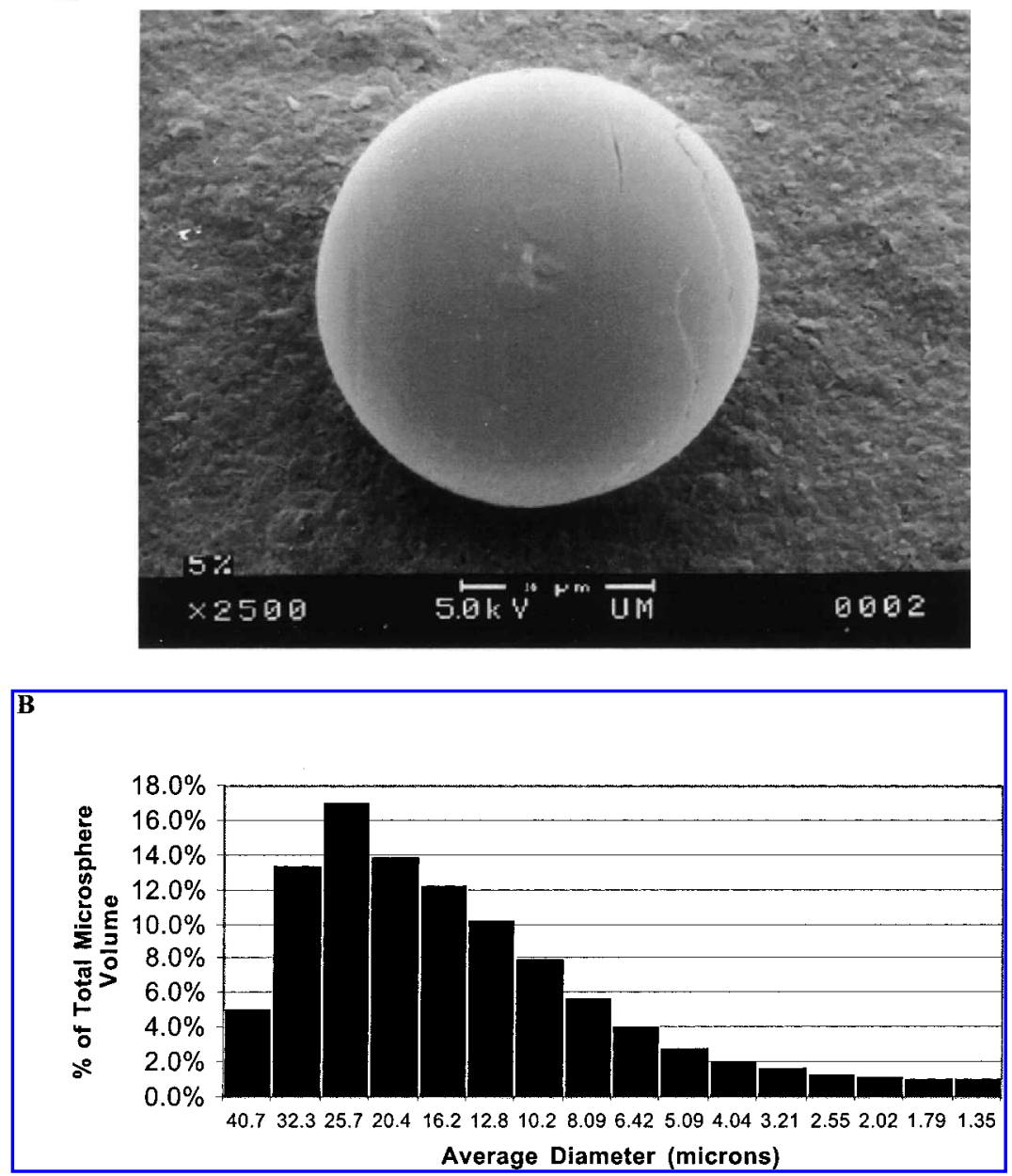

FIG. 1. Microsphere characterization.(A) Scanning electron micrograph of a PLG microsphere, original magnification, $\times 2500$. (B) Size distribution of PLG microspheres as a function of each size's fraction of the total microsphere volume. 85:15 PLG microspheres demonstrate a number average particle diameter of $2.3 \mu \mathrm{m}$, and a volume average diameter of $20 \mu \mathrm{m}$. 

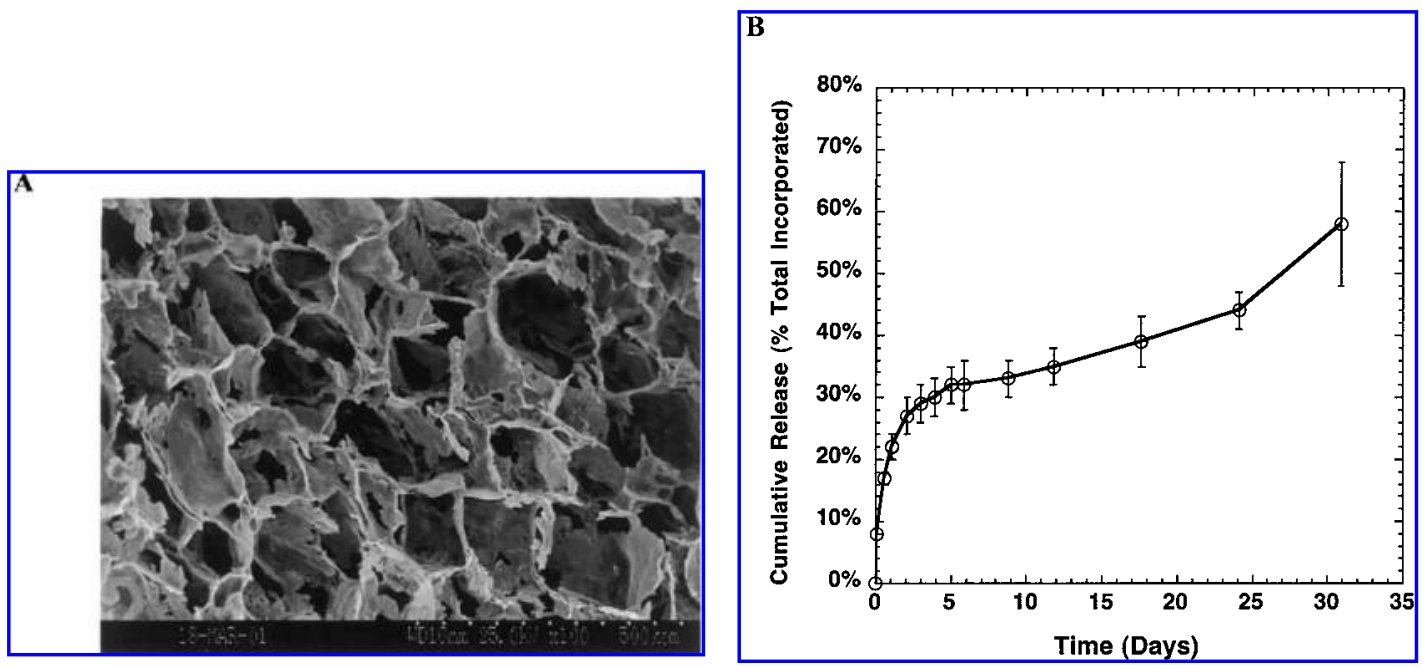

FIG. 2. Matrix characterization. (A) Scanning electron micrograph of PLG microsphere matrices containing $5 \mathrm{wt} \%$ alginate; original magnification, $\times 100$. (B) VEGF release from PLG matrices. Values represent means and standard deviation $(n=6)$.

SCID mice for a period of 7 or 14 days to evaluate the ability of delivered VEGF to enhance matrix vascularization. On retrieval the samples were stained for the presence of murine CD31 antigen to identify capillary blood vessels. A greater blood vessel density was present in VEGF-containing scaffolds than in control scaffolds at both time points. The enhanced vascularity was more pronounced at the 7-day time point (Fig. 3A and C) than at 14 days, as vessel density under all conditions had increased over time (Fig. 3B and D). Quantification confirmed these observations, as blood vessel density was nearly an order of magnitude greater in VEGF-releasing samples after 7 days, as compared with control samples (Fig. 4). After 14 days the VEGF conditions were ob-

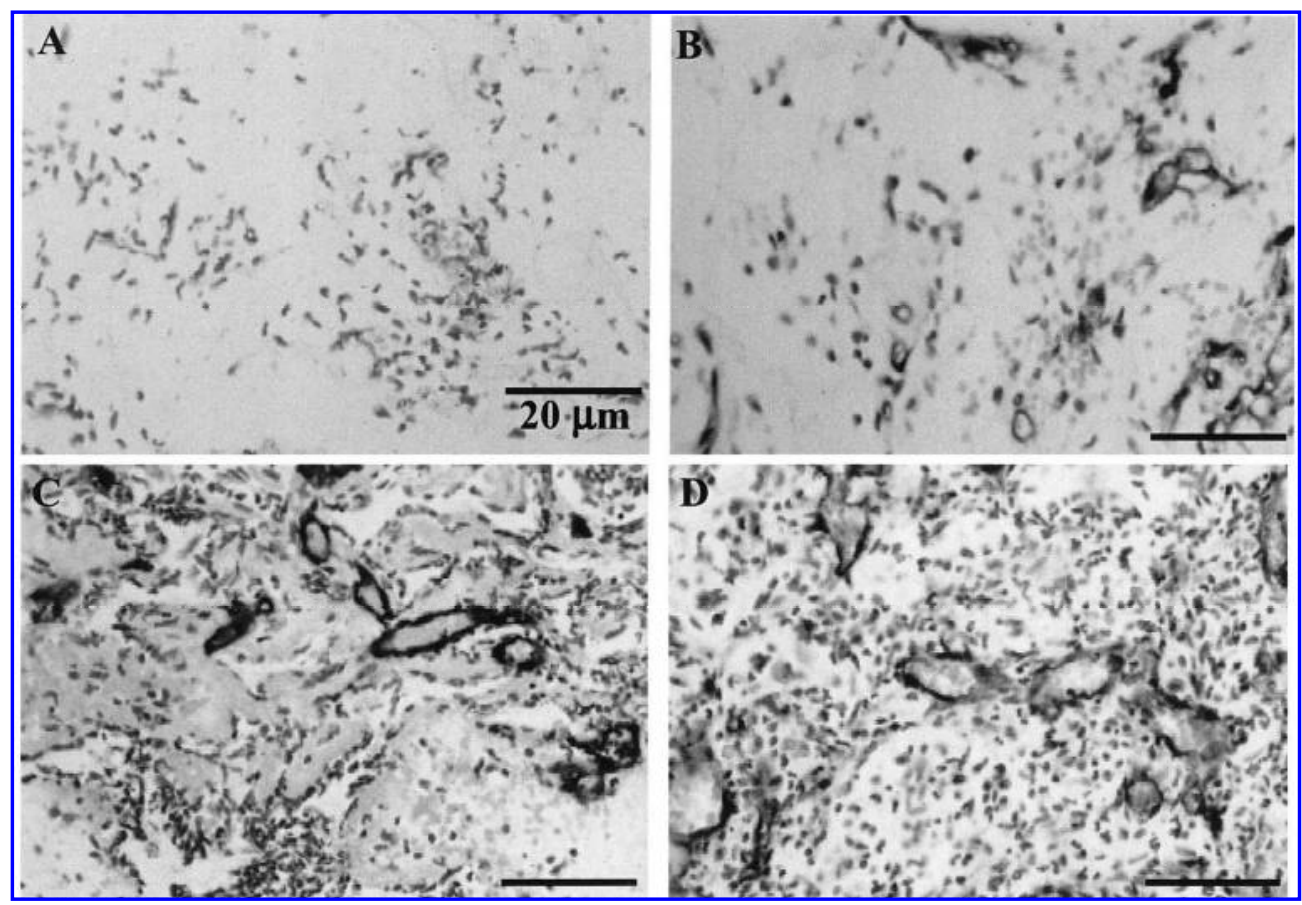

FIG. 3. VEGF delivery enhances blood vessel formation in implanted matrices as observed in tissue sections immunostained for mouse CD31 antigen. Blood vessels can be observed within the matrices as early as 7 days [(A) control; (C) VEGF] after implantation. After both 7 days and 14 days [(B control; (D) VEGF] many more positively stained vessels are present under VEGF-releasing conditions than under control conditions without growth factor. Original magnification, $\times 400$. 


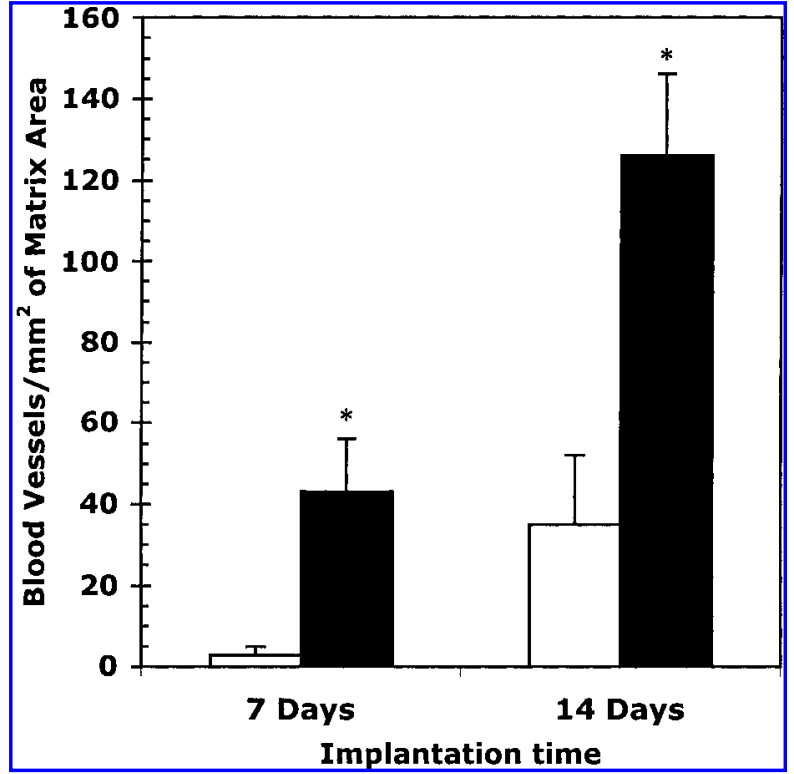

FIG. 4. Density of blood vessels in PLG matrices implanted into SCID mice. Blood vessels were manually counted at $\times 200$ magnification in tissue sections stained with antibodies for the presence of mouse CD31. Samples included control matrices without any growth factor (open columns) and matrices releasing VEGF (solid columns). The number of blood vessels counted in VEGF samples was significantly greater $(p<0.05)$ than that observed in control samples after 7 and 14 days. Results are expressed as mean values plus SEM $(n=3)$. served to yield approximately twice the number of blood vessels as control samples, although no qualitative differences in vessel diameter were observed between VEGF and control conditions.

\section{Hepatocyte survival}

To determine whether this method of improving vascularization would enhance the local environment and promote survival of transplanted cells, primary rat hepatocytes were seeded onto either VEGF-containing or blank control matrices and delivered subcutaneously to SCID mice. Hepatocytes were seeded with an efficiency of $53 \pm 8 \%$, and their survival was assessed 3 and 7 days after implantation. Observation of hematoxylin and eosin (H\&E)-stained sections revealed that the majority of hepatocytes under both VEGF and control conditions remained alive after 3 days in vivo (Fig. 5A and C). However, a difference in viable hepatocyte number became apparent at the day 7 time point. The total number of hepatocytes existing in control scaffolds had declined, and many of the remaining cells lacked evidence of a nucleus, indicating a loss of viability. Hepatocytes exhibiting a healthy-appearing morphology were more prevalent in VEGF-releasing samples, where overall hepatocyte density appeared similar to day 3 (Fig. 5B and D), and the majority of these surviving cells were located near ingrowing blood vessels. Quantification of transplanted cell

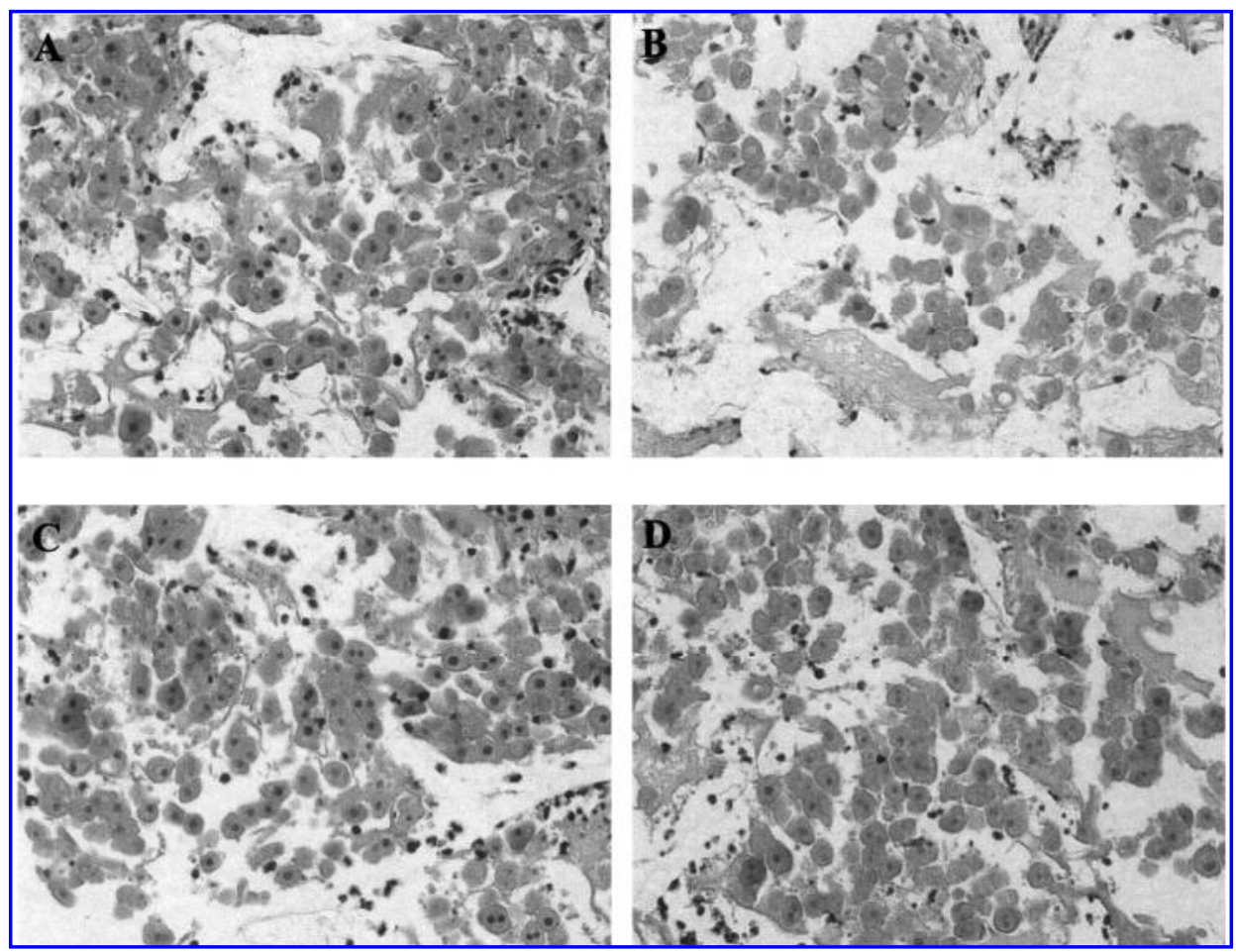

FIG. 5. H\&E-stained sections of matrices used for hepatocyte transplantation. Hepatocytes were seeded onto matrices, implanted into SCID mice, and retrieved after 3 days [(A) control; (C) VEGF] and 7 days [(B) control; (D) VEGF] days. Original magnification, $\times 400$. 
survival confirmed these observed trends, as there was no measurable difference between VEGF and control conditions in the fraction of hepatocytes surviving after 3 days in vivo, with approximately $70 \%$ of cells in the matrices remaining alive. To clearly indicate the significantly enhanced hepatocyte survival measured under VEGF-releasing conditions after 7 days $(p<0.05)$, the number of living cells at this time point has been normalized to the number of living cells on day 3 (Fig. 6). The number of hepatocytes surviving under VEGF conditions remained essentially constant from day 3 to day 7 , whereas survival of their counterparts under control conditions declined to less than $50 \%$ of the day 3 value during the same time interval.

To confirm that simultaneous hepatocyte delivery did not alter matrix vascularity, histological sections of matrices retrieved 7 days postimplantation were stained for CD31 to quantify induced blood vessel density, and the values were statistically similar to those for matrices implanted without cells (VEGF, $24 \pm 10$ vessels $/ \mathrm{mm}^{2}$; control, $2 \pm 1$ vessels $/ \mathrm{mm}^{2}$ ).

\section{DISCUSSION}

The results of this study indicate that sustained, localized delivery of VEGF from a polymer matrix enhances

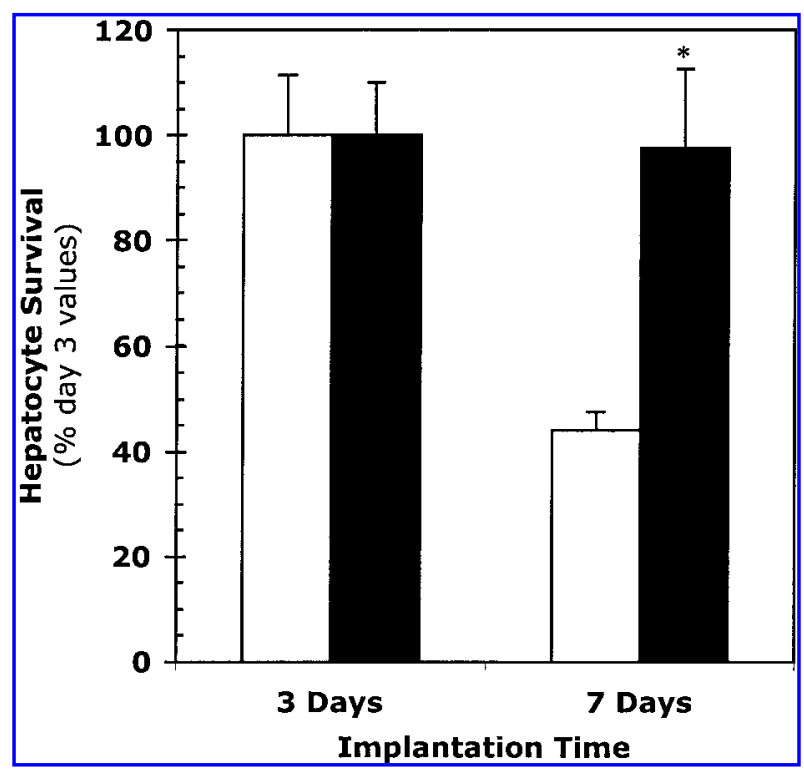

FIG. 6. Quantification of hepatocyte survival after transplantation. Hepatocytes were counted in histological sections at $\times 200$ magnification after 3 and 7 days in vivo. Data presented are normalized to the number of live cells present under each condition on day 3. On day 3, hepatocytes under control (open columns) and VEGF (solid columns) conditions exhibited approximately equal hepatocyte viability. However, VEGF-releasing matrices exhibited significantly greater hepatocyte survival at the day 7 time point as compared with control $(p<$ $0.05)$. Results are expressed as mean values plus SEM for two separate experiments, each with $n=3$. the formation of blood vessels within the construct, and that the extent of vascularization is similar with and without simultaneous delivery of cells. In addition, enhancing vascularization of the device through VEGF delivery promotes the survival of these transplanted cells. This system may find utility in cell-based therapies, and may be extended to directly manipulate the transplanted cells through delivery of additional growth factors specific to the cells of interest.

Delivery of VEGF from a cell transplantation matrix can significantly enhance blood vessel formation in the matrix. This significant enhancement of vascularization in VEGF-releasing matrices over the course of 2 weeks was anticipated from previous studies. ${ }^{15,25}$ However, in these earlier studies blood vessels were identified by their morphology in hematoxylin and eosin-stained sections. Here we have demonstrated that definitive blood vessel identification via endothelial cell-specific CD31 immunostaining gives trends similar to those determined by hematoxylin and eosin staining. The values for the measured vessel densities, under both VEGF and control conditions, were lower in this study than in our previous reports. This difference is related to a modification of the scaffold fabrication process. Matrices used in earlier VEGF delivery studies were composed of granular PLG, as opposed to the PLG microspheres used here, and when these granular-based matrices were sectioned for histological analysis the center portion of each section often became damaged or lost completely. Blood vessel number could then be quantified only in outer regions of the matrices, which are more heavily vascularized. This led to inflation of values for blood vessel density. Matrices formed with microspheres, on the other hand, retain their integrity during histological processing, making them superior for studies of vascularization as they allow for more accurate quantification of blood vessels throughout the entire section.

The increased blood vessel density resulting from VEGF delivery was clearly greater than under control conditions at the 1- and 2-week time points. The trend observed for induction of vessel growth is not surprising because a majority of the incorporated VEGF is released within 2 weeks, and the majority of that release occurs in the first 2-3 days. This result is also desirable, as our aim is to rapidly modify the environment within the matrix and then cease to influence the system so that a natural tissue, in physiologic balance with the host, can develop. Employing a localized delivery system for the growth factor generally minimizes the overall effect on the host, as it allows for enhancing blood vessel formation where necessary, while eliminating the risk of toxic side effects ${ }^{26}$ and unwanted blood vessels developing at other sites in the body. Localized, as opposed to systemic, delivery also makes it possible to deliver smaller amounts of growth factor to elicit the desired response. Sustained release is a significant aspect of the type of system pre- 
sented here, as previous studies with this system have demonstrated that bolus injection of an equivalent amount of VEGF into a blank matrix at the time of implantation leads to no significant improvement in vascularization. ${ }^{16}$ Importantly, when hepatocytes were transplanted on VEGF-releasing matrices, the trend of enhanced blood vessel ingrowth was essentially identical to that observed when cells were not included. Thus, the transplanted cell mass neither inhibited the ability of vessels to penetrate the matrix, nor produced enough VEGF to further enhance the vascular response. These are important findings, indicating that this system for promoting vascularization can be utilized in conjunction with cell delivery, and that it provides an added stimulus beyond that produced by the cells themselves. Differences in blood vessel diameter between VEGF and control conditions were not observed. This is not surprising, as VEGF is primarily an inducer of vascularization and other factors are likely required to promote formation of larger and more mature vessels. ${ }^{16}$

Enhanced vascularization induced by VEGF delivery correlated with significantly greater survival of implanted hepatocytes after 1 week, as compared with control conditions under which VEGF was not simultaneously delivered with the cells. There was not a detectable difference in hepatocyte survival at an intermediate 3-day time point, but a difference was not necessarily expected then, as the growth factor would not be expected to enhance new blood vessel ingrowth through the majority of the scaffold over this initial interval. However, after 7 days in vivo, the percentage of surviving hepatocytes under VEGF-releasing conditions was approximately twice that of hepatocytes implanted on blank control matrices. Other investigators have similarly demonstrated that increasing the vascularity of the transplant area, before hepatocyte implantation, can enhance engraftment of the cells, ${ }^{17,24,27,28}$ as can implantation in highly vascularized sites such as the small intestinal mesentery. ${ }^{18,29}$ Utilizing VEGF as a proangiogenic stimulus may additionally enhance oxygen delivery to the cells, because of the vasodilation it induces and the leakiness of vessels exposed to VEGF. Together, the results of our current report and others clearly illustrate the value of using proangiogenic interventions in conjunction with hepatocyte transplantation.

In this work, we enhanced transplanted hepatocyte survival indirectly, by delivering VEGF to manipulate the response of endothelial cells surrounding the implanted construct. Previous studies have demonstrated that surgical interventions (e.g., partial hepatectomy, portal-caval shunting) can also dramatically enhance hepatocyte engraftment and proliferation, ${ }^{29,30}$ and these processes may directly influence hepatocyte survival by altering the availability of blood-borne growth factors for hepatocytes. These surgical procedures could potentially be replaced by using the system described in this article for the controlled delivery of survival factors present in the portal circulation that act directly to enhance hepatocyte survival and function (e.g., epidermal growth factor, hepatocyte growth factor). ${ }^{2}$ In the context of the current study, delivery of these factors may prove particularly important in promoting survival during the initial days after transplantation, before VEGF is able to promote vascularization at the implant site. Sequences of growth factors, ${ }^{16}$ or plasmid DNA, ${ }^{31}$ can also be readily delivered with this system.

In summary, we have developed a system capable of simultaneously delivering growth factors and cell populations with potential therapeutic applications. This system may be utilized in future studies with other growth factors, or combinations of growth factors, and other cell types to enhance the survival and function of transplanted cells.

\section{ACKNOWLEDGMENTS}

The authors thank Alessandra Ennett and Kathryn Riddle for assistance with implant surgeries. Financial support for this work was provided by the National Institutes of Health (R01 DE 13349). M.C.P. was supported by a Whitaker Foundation fellowship, M.K.S. was supported by an NIH Cellular Biotechnology Training Program fellowship, and T.P.R. was supported by the National Institute of Dental and Craniofacial Research (T32 DE 07057).

\section{REFERENCES}

1. Davis, M.W., and Vacanti, J.P. Toward development of an implantable tissue engineered liver. Biomaterials 17, 365, 1996.

2. Mooney, D.J., Park, S., Kaufmann, P.M., Sano, K., McNamara, K., Vacanti, J.P., and Langer, R. Biodegradable sponges for hepatocyte transplantation. J. Biomed. Mater. Res. 29, 959, 1995.

3. Carmeliet, P., and Jain, R.K. Angiogenesis in cancer and other diseases. Nature 407, 249, 2000.

4. Colton, C.K. Implantable biohybrid artificial organs. Cell Transplant. 4, 415, 1995.

5. Beck, L., and D'Amore, P.A. Vascular development: Cellular and molecular regulation. FASEB J. 11, 366, 1997.

6. Ferrara, N. Vascular endothelial growth factor. Eur. J. Cancer 32A, 2413, 1996.

7. Yancopoulos, G.D., Davis, S., Gale, N.W., Rudge, J.S., Wiegand, S.J., and Holash, J. Vascular-specific growth factors and blood vessel formation. Nature 407, 242, 2000.

8. Polverini, P.J. The pathophysiology of angiogenesis. Crit. Rev. Oral Biol. Med. 6, 230, 1995. 
9. Gombotz, W.R., and Pettit, D.K. Biodegradable polymers for protein and peptide drug delivery. Bioconjug Chem $\mathbf{6}$, 332, 1995.

10. Mooney, D.J., Kaufmann, P.M., Sano, K., Schwendeman, S.P., Majahod, K., Schloo, B., Vacanti, J.P., and Langer, R. Localized delivery of epidermal growth factor improves the survival of transplanted hepatocytes. Biotechnol. Bioeng. 50, 422, 1996.

11. Cleland, J.L. Solvent evaporation processes for the production of controlled release in biodegradable microsphere formulations for therapeutics and vaccines. Biotechnol. Prog. 14, 102, 1998.

12. Eiselt, P., Kim, B.S., Chacko, B., Isenberg, B., Peters, M.C., Greene, K.G., Roland, W.P., Loebsack, A.B., Burg, K.J., Culberson, C., Halberstadt, C.R., Holder, W.D., and Mooney, D.J. Development of technologies aiding largetissue engineering. Biotechnol. Prog. 14, 134, 1998.

13. King, T.W., and Patrick, C.W., Jr. Development and in vitro characterization of vascular endothelial growth factor (VEGF)-loaded poly(DL-lactic-co-glycolic acid)/poly (ethylene glycol) microspheres using a solid encapsulation/single emulsion/solvent extraction technique. J. Biomed. Mater. Res. 51, 383, 2000.

14. Murphy, W.L., Peters, M.C., Kohn, D.H., and Mooney, D.J. Sustained release of vascular endothelial growth factor from mineralized poly(lactide-co-glycolide) scaffolds for tissue engineering. Biomaterials 21, 2521, 2000.

15. Lee, K.Y., Peters, M.C., Anderson, K.W., and Mooney, D.J. Controlled growth factor release from synthetic extracellular matrices. Nature 408, 998, 2000.

16. Richardson, T.P., Peters, M.C., Ennett, A.B., and Mooney, D.J. Polymeric system for dual growth factor delivery. Nat. Biotechnol. 19, 1029, 2001.

17. Elcin, Y.M., Dixit, V., Lewin, K., and Gitnick, G. Xenotransplantation of fetal porcine hepatocytes in rats using a tissue engineering approach. Artif. Organs 23, 146, 1999.

18. Mooney, D.J., Sano, K., Kaufmann, P.M., Majahod, K., Schloo, B., Vacanti, J.P., and Langer, R. Long-term engraftment of hepatocytes transplanted on biodegradable polymer sponges. J. Biomed. Mater. Res. 37, 413, 1997.

19. Mooney, D.J., Baldwin, D.F., Suh, N.P., Vacanti, J.P., and Langer, R. Novel approach to fabricate porous sponges of poly(D,L-lactic-co-glycolicacid) without the use of organic solvents. Biomaterials 17, 1417, 1996.

20. Richardson, T.P., and Mooney, D.J. Gas foam processing for tissue engineering applications. In: Atala, A., and Lanza, R., eds. Methods of Tissue Engineering. San Diego, CA: Academic Press, 2001, pp. 653-662.

21. Cohen, S., Yoshioka, T., Lucarelli, M., Hwang, L.H., and Langer, R. Controlled delivery systems for proteins based on poly(lactic/glycolic acid) microspheres. Pharmacol. Res. 8, 713, 1991.

22. Aiken, J., Cima, L., Schloo, B., Mooney, D., Johnson, L., Langer, R., and Vacanti, J.P. Studies in rat liver perfusion for optimal harvest of hepatocytes. J. Pediatr. Surg. 25, 140, 1990.

22a. Institute of Laboratory Animal Resources. Guide for the Care and Use of Laboratory Animals. NIH Publication 8523. Bethesda, MD: National Institutes of Health, 1985.

23. Jaffe, V., Darby, H., Bishop, A., and Hodgson, H.J. The growth of liver cells in the pancreas after intra-splenic implantation: The effects of portal perfusion. Int. J. Exp. Pathol. 72, 289, 1991.

24. Lee, H., Cusick, R.A., Browne, F., Kim, T.H., Ma, P.X., Utsunomiya, H., Langer, R., and Vacanti, J.P. Local delivery of basic fibroblast growth factor increases both angiogenesis and engraftment of hepatocytes in tissue-engineered polymer devices. Transplantation 73, 1589, 2002.

25. Peters, M.C., Polverini, P.J., and Mooney, D.J. Engineering vascular networks in porous polymer matrices. J. Biomed. Mater. Res. 60, 668, 2002.

26. Mazue, G., Newman, A.J., Scampini, G., Della Torre, P., Hard, G.C., Iatropoulos, M.J., Williams, G.M., and Bagnasco, S.M. The histopathology of kidney changes in rats and monkeys following intravenous administration of massive doses of FCE 26184, human basic fibroblast growth factor. Toxicol. Pathol. 21, 490, 1993.

27. Ajioka, I., Akaike, T., and Watanabe, Y. Expression of vascular endothelial growth factor promotes colonization, vascularization and growth of transplanted hepatic tissues in the mouse. Hepatology 29, 396, 1999.

28. Aijoka, I., Nishio, R., Ikekita, M., Akaike, T., Saskai, M., Enami, J., and Watanabe, Y. Establishment of heterotropic liver tissue mass with direct link to the host liver following implantation of hepatocytes transfected with vascular endothelial growth factor gene in mice. Tissue Eng. 7, 335, 2001.

29. Kneser, U., Kaufmann, P.M., Fiegel, H.C., Pollok, J.M., Kluth, D., Herbst, H., and Rogiers, X. Long-term differentiated function of heterotopically transplanted hepatocytes on three-dimensional polymer matrices. J. Biomed. Mater. Res. 47, 494, 1999.

30. Kaufmann, P.M., Sano, K., Breuer, C.K., Organ, G.M., Schloo, B.L., Kluth, D., and Vacanti, J.P. Evaluation of methods of hepatotrophic stimulation in rat heterotopic hepatocyte transplantation using polymers. J. Pediatr. Surg. 34, 1118, 1999.

31. Shea, L.D., Smiley, E., Bonadio, J., and Mooney, D.J. DNA delivery from polymer matrices for tissue engineering. Nat. Biotechnol. 17, 551, 1999.

Address reprint requests to: David J. Mooney, Ph.D.

5213 Dental School 1011 N. University Ave Ann Arbor, MI 48109-1078

E-mail: mooneyd@umich.edu 


\section{This article has been cited by:}

1. Sung Mook Lim, Hyo Jung Lee, Se Heang Oh, Jin Man Kim, Jin Ho Lee. 2009. Novel fabrication of PCL porous beads for use as an injectable cell carrier system. Journal of Biomedical Materials Research Part B: Applied Biomaterials 90B:2, 521-530. [CrossRef]

2. Cristiano Spadaccio, Massimo Chello, Marcella Trombetta, Alberto Rainer, Yoshiya Toyoda, Jorge A. Genovese. 2009. Drug releasing systems in cardiovascular tissue engineering. Journal of Cellular and Molecular Medicine 13:3, 422-439. [CrossRef]

3. Jake Barralet, Uwe Gbureck, Pamela Habibovic, Elke Vorndran , Catherine Gerard, Charles J. Doillon . Angiogenesis in Calcium Phosphate Scaffolds by Inorganic Copper Ion ReleaseAngiogenesis in Calcium Phosphate Scaffolds by Inorganic Copper Ion Release. Tissue Engineering Part A, ahead of print. [Abstract] [PDF] [PDF Plus]

4. Aylin Sendemir Urkmez, Sherrie G. Clark, Matthew B. Wheeler, Michael S. Goldwasser, Russell D. Jamison. 2008. Evaluation of Chitosan/Biphasic Calcium Phosphate Scaffolds for Maxillofacial Bone Tissue Engineering. Macromolecular Symposia 269:1, 100-105. [CrossRef]

5. Anming Xiong, Timothy W. Austin, Eric Lagasse, Nobuko Uchida, Stanley Tamaki, Bruno B. Bordier, Irving L. Weissman, Jeffrey S. Glenn, Maria T. Millan . 2008. Isolation of Human Fetal Liver Progenitors and Their Enhanced Proliferation by Three-Dimensional Coculture with Endothelial CellsIsolation of Human Fetal Liver Progenitors and Their Enhanced Proliferation by Three-Dimensional Coculture with Endothelial Cells. Tissue Engineering Part A 14:6, 995-1006. [Abstract] [PDF] [PDF Plus] [Supplementary material]

6. Anming Xiong, Timothy W. Austin, Eric Lagasse, Nobuko Uchida, Stanley Tamaki, Bruno B. Bordier, Irving L. Weissman, Jeffrey S. Glenn, Maria T. Millan. 2008. Isolation of Human Fetal Liver Progenitors and Their Enhanced Proliferation by Three-Dimensional Coculture with Endothelial Cells. Tissue Engineering Part $A$, ahead of print080423075413219. [CrossRef]

7. Alton M. Hiscox , Alice L. Stone, Sean Limesand, James B. Hoying, Stuart K. Williams . 2008. An Islet-Stabilizing Implant Constructed Using a Preformed VasculatureAn Islet-Stabilizing Implant Constructed Using a Preformed Vasculature. Tissue Engineering Part A 14:3, 433-440. [Abstract] [PDF] [PDF Plus]

8. Vladislav Tsiperson, Orit Goldshmidt, Neta Ilan, Gideon Shoshany, Israel Vlodavsky, Ella Veitsman , Yaacov Baruch . 2008. Heparanase Enhances Early Hepatocyte Inclusion in the Recipient Liver after Transplantation in Partially Hepatectomized RatsHeparanase Enhances Early Hepatocyte Inclusion in the Recipient Liver after Transplantation in Partially Hepatectomized Rats. Tissue Engineering Part A 14:3, 449-458. [Abstract] [PDF] [PDF Plus]

9. Alton M. Hiscox , Alice L. Stone, Sean Limesand, James B. Hoying , Stuart K. Williams . An Islet-Stabilizing Implant Constructed Using a Preformed VasculatureAn Islet-Stabilizing Implant Constructed Using a Preformed Vasculature. Tissue Engineering, ahead of print. [Abstract] [PDF] [PDF Plus]

10. Vladislav Tsiperson, Orit Goldshmidt, Neta Ilan, Gideon Shoshany, Israel Vlodavsky, Ella Veitsman , Yaacov Baruch. Heparanase Enhances Early Hepatocyte Inclusion in the Recipient Liver after Transplantation in Partially Hepatectomized RatsHeparanase Enhances Early Hepatocyte Inclusion in the Recipient Liver after Transplantation in Partially Hepatectomized Rats. Tissue Engineering, ahead of print. [Abstract] [PDF] [PDF Plus]

11. Jennifer J. Vallbacka, Michael V. Sefton . 2007. Vascularization and Improved In Vivo Survival of VEGF-Secreting Cells Microencapsulated in HEMA-MMAVascularization and Improved In Vivo Survival of VEGF-Secreting Cells Microencapsulated in HEMA-MMA. Tissue Engineering 13:9, 2259-2269. [Abstract] [PDF] [PDF Plus] 
12. Mark J. Butler, Michael V. Sefton. 2007. Poly(butyl methacrylate-co-methacrylic acid) tissue engineering scaffold with pro-angiogenic potentialin vivo. Journal of Biomedical Materials Research Part A 82A:2, 265-273. [CrossRef]

13. Kathryn W Riddle, Hyun-Joon Kong, J Kent Leach, Claudia Fischbach, Charles Cheung, Kristi S Anseth, David J Mooney. 2007. Modifying the Proliferative State of Target Cells to Control DNA Expression and Identifying Cell Types Transfected In Vivo. Molecular Therapy 15:2, 361-368. [CrossRef]

14. Antonios G. Mikos, Susan W. Herring, Pannee Ochareon, Jennifer Elisseeff, Helen H. Lu, Rita Kandel , Frederick J. Schoen, Mehmet Toner, David Mooney, Anthony Atala , Mark E. Van Dyke, David Kaplan , Gordana Vunjak-Novakovic . 2006. Engineering Complex TissuesEngineering Complex Tissues. Tissue Engineering 12:12, 3307-3339. [Abstract] [PDF] [PDF Plus]

15. Kristen A. Wieghaus, Scott M. Capitosti, Christopher R. Anderson, Richard J. Price, Brett R. Blackman, Milton L. Brown, Edward A. Botchwey. 2006. Small Molecule Inducers of Angiogenesis for Tissue Engineering. Tissue Engineering, ahead of print060706073730072. [CrossRef]

16. Kristen A. Wieghaus, Scott M. Capitosti, Christopher R. Anderson, Richard J. Price, Brett R. Blackman , Milton L. Brown , Edward A. Botchwey . 2006. Small Molecule Inducers of Angiogenesis for Tissue EngineeringSmall Molecule Inducers of Angiogenesis for Tissue Engineering. Tissue Engineering 12:7, 1903-1913. [Abstract] [PDF] [PDF Plus]

17. Zigang Ge, Fang Yang, James C. H. Goh, Seeram Ramakrishna, Eng Hin Lee. 2006. Biomaterials and scaffolds for ligament tissue engineering. Journal of Biomedical Materials Research Part A 77A:3, 639-652. [CrossRef]

18. Susan X. Hsiong, David J. Mooney. 2006. Regeneration of vascularized bone. Periodontology 2000 41:1, 109-122. [CrossRef]

19. Kristen A. Wieghaus, Scott M. Capitosti, Christopher R. Anderson, Richard J. Price, Brett R. Blackman, Milton L. Brown, Edward A. Botchwey. 2006. Small Molecule Inducers of Angiogenesis for Tissue Engineering. Tissue Engineering, ahead of print060802052515050. [CrossRef]

20. Molly K. Smith, Kathryn W. Riddle, David J. Mooney. 2006. Delivery of Hepatotrophic Factors Fails to Enhance Longer-Term Survival of Subcutaneously Transplanted Hepatocytes. Tissue Engineering, ahead of print060303085351001. [CrossRef]

21. A. Eser Elcin , Y. Murat Elcin . 2006. Localized Angiogenesis Induced by Human Vascular Endothelial Growth Factor-Activated PLGA SpongeLocalized Angiogenesis Induced by Human Vascular Endothelial Growth Factor-Activated PLGA Sponge. Tissue Engineering 12:4, 959-968. [Abstract] [PDF] [PDF Plus]

22. Molly K. Smith , Kathryn W. Riddle , David J. Mooney . 2006. Delivery of Hepatotrophic Factors Fails to Enhance Longer-Term Survival of Subcutaneously Transplanted HepatocytesDelivery of Hepatotrophic Factors Fails to Enhance Longer-Term Survival of Subcutaneously Transplanted Hepatocytes. Tissue Engineering 12:2, 235-244. [Abstract] [PDF] [PDF Plus]

23. T. Yokoyama, K. Ohashi, H. Kuge, H. Kanehiro, H. Iwata, M. Yamato, Y. Nakajima. 2006. In Vivo Engineering of Metabolically Active Hepatic Tissues in a Neovascularized Subcutaneous Cavity. American Journal of Transplantation 6:1, 50-59. [CrossRef]

24. Alon Kedem, Anat Perets, Iris Gamlieli-Bonshtein, Mona Dvir-Ginzberg, Solly Mizrahi, Smadar Cohen . 2005. Vascular Endothelial Growth Factor-Releasing Scaffolds Enhance Vascularization and Engraftment of Hepatocytes Transplanted on Liver LobesVascular Endothelial Growth Factor-Releasing Scaffolds Enhance Vascularization and Engraftment of Hepatocytes Transplanted on Liver Lobes. Tissue Engineering 11:5-6, 715-722. [Abstract] [PDF] [PDF Plus]

25. Ya-Chun Huang, Chiung-Tong Chen, Sung-Ching Chen, Po-Hong Lai, Huang-Chien Liang, Yen Chang, Lin-Chien Yu, Hsing-Wen Sung. 2005. A Natural Compound (Ginsenoside Re) Isolated from Panax ginseng as a Novel Angiogenic Agent for Tissue Regeneration. Pharmaceutical Research 22:4, 636-646. [CrossRef]

26. J Kent Leach, David J Mooney. 2004. Bone engineering by controlled delivery of osteoinductive molecules and cells. Expert Opinion on Biological Therapy 4:7, 1015-1027. [CrossRef] 